\title{
Prevalencia de la depresión post parto en Arequipa, Perú
}

\author{
Prevalence of postpartum depression in Arequipa, Peru \\ María Alejandra Masías Salinas ${ }^{1, a}$, Walter L. Arias Gallegos ${ }^{1, b}$

\section{RESUMEN}

Objetivos: Determinar la prevalencia de la depresión postparto en mujeres puérperas de la ciudad de Arequipa. Material y métodos: Estudio descriptivo. Se evaluaron a 113 madres que acudieron a sus controles postnatales en una posta de salud de la ciudad. Se utilizó la Escala de Depresión Postparto de Edimburgo, previo consentimiento informado. Para efectos del estudio, se valoraron las propiedades psicométricas del instrumento, reportándose adecuados niveles de validez y confiabilidad para la muestra evaluada. Resultados: Los resultados indican que aproximadamente, el $41 \%$ de las madres no tiene riesgo de depresión postparto, $14 \%$ presenta riesgo y $45 \%$ tiene síntomas de depresión postparto. Asimismo, se encontró que el grado de instrucción se relaciona de manera negativa con los niveles de depresión postparto, pero no hubo diferencias significativas en función del tipo de parto ni el estado civil de las madres. Conclusiones: Se concluye que el $45 \%$ de las mujeres puérperas evaluadas tienen síntomas de depresión postparto y que el grado de instrucción presenta correlaciones negativas con esta variable.

PALABRAS CLAVE: Depresión postparto, puérperas, familia. (Fuente: DeCS Bireme).

\section{SUMMARY}

Objectives: To determine the prevalence of postpartum depression in puerperal women in the city of Arequipa. Methods: A descriptive study was conducted in 113 puerperal women that attended peripheral health care centers for postpartum control. Edinburgh scale for depression was used after getting inform consent. Psychometric properties of the tool were evaluated. Results: Our results indicate that $41 \%$ of mothers are not at risk of postpartum depression; $14 \%$ are at risk and $45 \%$ have symptoms of postpartum depression; the degree of education was inversely correlated with postpartum depression and we did not find statistical association with route of delivery and marital status. Conclusions: We conclude that $45 \%$ of puerperal women evaluated had depression and that the degree of education inversely correlated with it.

KEYWORDS: Depression postpartum, parturient women, family. (Source: MeSH NLM).

\section{INTRODUCCIÓN}

El parto es una etapa de la gestación que implica una serie de cambios físicos, psicológicos y sociales para la mujer. A nivel físico, se han descrito, cambios estructurales del cerebro de las madres, que implican un aumento del volumen de la sustancia gris en el córtex prefrontal superior, medio e inferior, así como en el lóbulo parietal, el hipotálamo y la amígdala (1). Estos cambios se asocian con la percepción positiva que tienen las madres de sus bebés y un incremento del arousal que las mantiene alertas ante las demandas de sus hijos. Sin embargo, estos cambios no se presentan en madres que no desarrollan conductas de cuidado

\footnotetext{
Escuela Profesional de Psicología, Universidad Católica San Pablo. Arequipa, Perú.

Licenciada en Psicología;

Psicólogo; Maestro en Ciencias de la Educación; Profesor auxiliar y docente investigador
} 
de sus bebés (1). De hecho, las madres que han estado expuestas a estresores de manera prolongada durante la infancia, pueden tener niveles anormales de dopamina y oxitocina (2).

Psicológicamente, el parto conlleva un elevado nivel de estrés, que puede degenerar en trastornos adaptativos, trastornos de personalidad y trastornos del estado del ánimo (3). Según Jadresic (4), más del $40 \%$ de las mujeres sufre de síntomas depresivos y ansiosos inespecíficos, y entre un $10 \%$ y $15 \%$ sufren trastornos depresivos propiamente dichos. La depresión postparto es el desarrollo de depresión en la madre luego del nacimiento de su hijo, y se caracteriza por llanto, desánimo, sentimientos de incapacidad para enfrentar el rol de madre, y suele durar entre 6 y 8 semanas, o llegar incluso hasta un año (5). Este estado, puede afectar profundamente a la madre, al bebé, su relación de pareja y a la familia nuclear o extendida (6). A pesar de lo grave en que se puede tornar la depresión postparto, solo el $20 \%$ recibe tratamiento, debido a que, en muchos casos, la madre no la reconoce como un problema (7).

Socialmente, la depresión postparto, puede estar mediada por una serie de factores. Uno de ellos, es la edad de la madre, por ejemplo, las madres adolescentes no han madurado lo suficiente como para afrontar su rol de madre, lo que les genera angustia (8). Asimismo, otros estudios han señalado que las familias monoparentales implican mayores riesgos para la estabilidad emocional de la madre y la atención de las necesidades de los hijos (9). Por otro lado, el nivel socioeconómico de la madre, se ha asociado con el apego materno inseguro (10). En ese sentido, el apoyo emocional recibido de los padres en la infancia, se asocia con la salud física y psicológica de los hijos (11); por lo que, madres que no han recibido un soporte emocional parental sólido, están más propensas a sufrir de depresión postparto.

De hecho, la depresión postparto, puede tener un impacto negativo en el desarrollo del bebé, ya que las madres que se perciben como no eficaces en su rol maternal, tienden a adoptar prácticas parentales negativas (12), aunque tales prácticas pueden verse influidas por diferencias culturales sobre el rol de madre y la interacción con los hijos (13).

En general, el estrés y los antecedentes psicosociales de la madre, son los factores que mejor explican la depresión postparto (14). Por ejemplo, en un estudio con 20 madres y sus bebés, se reportó que, aquellas que tenían menos estrés podían regular mejor el estado de ánimo de sus hijos, pero los bebés de las madres más estresadas experimentaron reacciones maternas menos sintonizadas y reguladoras de su estado emocional (15).

En ese sentido, la relación madre-hijo está influida mutuamente, porque si bien la madre atiende las necesidades del bebé, la aparición de nuevas conductas por parte de este puede generar la emergencia de nuevas conductas de parte de la madre (16). Asimismo, como ya se dijo, los niños de madres con síntomas depresivos están en riesgo de tener un pobre desarrollo psicosocial, con una afectividad negativa característica y falta de iniciativa y compromiso. Esto debido a que han desarrollado un apego inseguro (17). En el estudio cualitativo de Traverso con dos madres adolescentes peruanas, se encontró que aquella que tuvo una mejor relación con su madre, tenía una actitud más favorable hacia su bebé, mientras que la madre que tuvo una relación empobrecida con su madre, tenía mayores dificultades para hacerse cargo de su propio bebé (18).

Los factores de riesgo de la depresión postparto incluyen, además, historia previa de depresión, falta de soporte social, pobreza, violencia familiar, dificultades en la relación de pareja, historia de aborto previo, ambivalencia en relación a la gestación y ansiedad con respecto al bebé (19). En ese sentido, las expectativas de la madre frente al nacimiento del bebé, son importantes, y aunque pueden contener dosis de ansiedad, miedo y nerviosismo (20), el contar con los soportes sociales, familiares y emocionales adecuados; permite afrontar mejor el momento del parto y asumir el rol de madre de manera adecuada.

Lamentablemente, en el Perú, las condiciones en que muchas mujeres enfrentan la maternidad son sumamente difíciles. Un alto porcentaje de mujeres que van a ser madres viven en condiciones precarias, no cuentan con estudios superiores ni soportes económicos estables. Tampoco provienen de hogares bien constituidos, sino que más bien, han sido testigos o víctimas de violencia intrafamiliar y no han contado con las atenciones pertinentes de parte de sus padres (21). El estudio, tuvo como objetivo estimar la prevalencia de la depresión postparto en la ciudad de Arequipa.

\section{MATERIAL Y MÉTODOS}

Estudio descriptivo. La muestra estuvo conformada por 113 madres puérperas (entre los 45 días y las 6 
semanas después del parto) que habían hecho sus controles y seguimiento en una posta de salud del distrito de Alto Selva Alegre de la ciudad de Arequipa. Los criterios de inclusión fueron ser madre puérpera, que asistían a sus controles en la posta de salud mencionada, que no tuvieran antecedentes psiquiátricos y que desearan participar voluntariamente en el estudio. El muestreo fue no probabilístico mediante la técnica de muestreo por cuotas (23).

En el $28,31 \%$ de las madres eran de estado civil casada, $23 \%$ soltera, $47,78 \%$ conviviente y el $0,88 \%$ divorciada. En cuanto al grado de instrucción, 5,3\% tenía estudios primarios, $58,4 \%$ estudios secundarios, $8,8 \%$ estudio superior técnico y $27,4 \%$ estudios universitarios. El parto fue eutócico en el $61,94 \%$ y en el 38,05\% cesárea.

El instrumento utilizado fue la Escala de Depresión Postparto de Edimburgo, creada por Cox et al en 1987, y validada para gestantes de Lima por Lam et al (24). La escala consta de 10 ítem con cuatro opciones de respuesta de 0 a 4 puntos. Un puntaje menor a 10 indica que no hay riesgo de depresión postparto, un puntaje entre 10 y 12 indica que hay riesgo de depresión, mientras que puntajes superiores a 12 indican que existen síntomas de una probable depresión postparto. Se reportaron índices de confiabilidad superiores a 0,7 y correlación ítem-test mayor de 0,45 , por lo que el instrumento puede considerarse como válido y confiable.

\section{Procedimiento}

Se realizaron las coordinaciones con las autoridades de la Posta de Salud de Alto Selva Alegre, para obtener los permisos correspondientes. Posteriormente, se informó a las puérperas durante sus citas correspondientes en el área de obstetricia, acerca de los fines del estudio y la importancia de su participación. Con su consentimiento se procedió a aplicar la Escala de Depresión de Postparto de Edimburgo. Este estudio fue aprobado por el Comité de Ética de la escuela de Psicología de la Universidad Católica San Pablo.

\section{Análisis de datos}

El análisis estadístico implicó en primer lugar, obtener lo valores de validez y confiabilidad para la muestra de estudio, pues eran mujeres de la ciudad de Arequipa, mientras que la versión utilizada del instrumento se validó en mujeres de Lima. Luego se procesaron los datos relativos a la depresión postparto a nivel descriptivo; se utilizó la prueba $t$ de student y el análisis de varianza, para determinar las diferencias entre el estado civil, el grado de instrucción y el tipo de parto. También se realizó correlación entre los datos de las variables continuas y ordinales.

\section{RESULTADOS}

Para determinar la validez del instrumento, se realizó correlación ítem-test con el coeficiente de correlación de Pearson, obteniéndose valores entre 0,373 y 0,801 , lo que indica que los ítems del instrumento son homogéneos. El análisis factorial para determinar la validez de constructo, arrojó un valor KMO de 0,860 y una prueba de esfericidad de Bartlett significativa $(\mathrm{X} 2=404,133 ; \mathrm{gl}=45, \mathrm{p}<0,000)$, lo que indica que la prueba puede someterse a factorización. Se aplicó así, el análisis factorial exploratorio mediante el método de componentes principales, encontrándose

Tabla 1. Estadísticos psicométricos.

\begin{tabular}{lcccc}
\hline Ítems & $\mathbf{M}$ & $\mathbf{D E}$ & Asimetría & ritc \\
\hline 1 & 0,566 & 0,625 & 0,638 & 0,373 \\
2 & 0,831 & 0,962 & 0,957 & 0,486 \\
3 & 1,557 & 1,093 & $-0,254$ & 0,749 \\
4 & 1,292 & 0,941 & $-0,162$ & 0,487 \\
5 & 1,371 & 1,062 & $-0,021$ & 0,712 \\
6 & 1,610 & 0,967 & $-0,229$ & 0,671 \\
7 & 1,398 & 1,065 & $-0,045$ & 0,801 \\
8 & 1,035 & 0,915 & 0,711 & 0,742 \\
9 & 1,008 & 0,949 & 0,619 & 0,757 \\
10 & 0,628 & 1,001 & 1,294 & 0,695 \\
Total & 11,30 & 6,338 & 0,059 & -- \\
Factores & $\mathbf{F} \mathbf{1}$ & $\mathbf{F 2}$ & & \\
Ítem 9 & 0,823 & & & \\
Ítem 10 & 0,797 & & & \\
Ítem 8 & 0,766 & & & \\
Ítem 3 & 0,732 & & & \\
Ítem 7 & 0,705 & & & \\
Ítem 5 & 0,656 & & & \\
Ítem 6 & 0,645 & & & \\
Ítem 1 & & 0,692 & & \\
Ítem 2 & & 0,681 & & \\
Ítem 4 & & 0,631 & & \\
$\alpha$ & 0,853 & 0,761 & & \\
\hline
\end{tabular}


dos factores que explican el $55,69 \%$ de la varianza total de la prueba. El primer factor agrupa los ítems negativos $(3,5,6,7,8,9,10)$ y explica el $38,52 \%$ de la varianza, mientras que el segundo factor agrupa los ítems positivos $(1,2,4)$ explicando el $17,16 \%$ de la varianza total. Asimismo, el índice de confiabilidad mediante el método de consistencia interna con la prueba Alfa de Cronbach, fue de 0,853. Por tanto, se puede afirmar que la prueba utilizada es válida y confiable para la muestra de estudio (Tabla 1).

En cuanto a la depresión postparto, se obtuvo una media de $11,3 \pm 6,33$. El $40,7 \%$ no presentaba riesgo de depresión postparto; $14,15 \%$ tenía riesgo y $45,13 \%$ presentaba síntomas de depresión postparto.

Para evaluar la relación entre depresión postparto y edad de la madre, se utilizó el coeficiente de correlación de Pearson, obteniéndose $\mathrm{r}=-0,038$, indicando que no existe relación entre estas variables. Para determinar la relación entre nivel de depresión y grado de instrucción, se utilizó la prueba Tau b de Kendall obteniéndose $\mathrm{r}=$ 0,212 , lo que indica que, a mayor grado de instrucción, menor será el nivel de depresión postparto, pero solo en el $20 \%$ de las mujeres evaluadas.

No se encontró diferencias significativas entre niveles de depresión postparto y parto natural o cesárea, aunque la media fue mayor en la que el parto fue por cesárea. Tampoco se encontró diferencia significativa entre depresión postparto en función del estado civil.

\section{DISCUSIÓN}

En el presente estudio se ha encontrado una elevada frecuencia de madres puérperas con síntomas de depresión postparto $(45,13 \%)$, siendo inclusive más elevado que el reportado en la ciudad de Lima, donde la prevalencia fue de $34,1 \%$ (24). Aunque no se encontró diferencia entre los niveles de depresión postparto y estado civil de las madres, se encontró que el grado de instrucción se relaciona negativamente con la depresión postparto. Esto podría sugerir, como se ha reportado en diversos estudios $(4,10,12,21)$, que el nivel socioeconómico es un factor determinante en la ocurrencia de la depresión postparto, ya que el grado de instrucción se encuentra fuertemente asociado al nivel socioeconómico.

Por otro lado, no se encontró diferencia en función del tipo de parto, a pesar de que algunos estudios señalan que las madres que han dado a luz por cesárea presentan niveles menores de oxitocina, mostrándose menos sensibles a las demandas de sus bebés, y con mayor riesgo de tener depresión postparto (1). Sin embargo, otros estudios señalan que aún en la cesárea, si se induce el parto con oxitocina, dicha práctica es beneficiosa tanto para la madre como para el bebé (2).

Ahora bien, el hecho de que las madres evaluadas durante el puerperio hayan tenido niveles altos de depresión postparto, merece especial atención por las implicancias que pueden tener en los neonatos. En ese sentido, aunque el parto suele ser valorado de manera negativa (20), hace falta enseñar a valorar la maternidad, pues también aporta al sentido de vida de la mujer, a través del enriquecimiento de su femineidad y su rol de madre (25). Para ello, es importante, desarrollar programas de intervención que brinden orientación a las madres después del parto y no solo antes, como ocurre con los talleres de profilaxis que desarrollan las obstetrices. También es importante que se estimule el fortalecimiento de los vínculos entre la madre y su bebé, a través de programas de intervención, especialmente diseñados para tales fines (26).

En tal sentido, sería importante retomar prácticas que servían de orientación para las madres durante las primeras décadas del siglo $\mathrm{XX}$, tales como la publicación de revistas especializadas, como la revista Madre y Niño, que se editó desde 1930 en Argentina (27); o la difusión de programas radiales que brinden orientación a las madres, como el que llevó a cabo Donald Winnicott entre 1939 y 1962, en Inglaterra (28). Asimismo, se debe promover un poco más el empoderamiento social de la mujer (29), ya que todavía es común ver casos de violencia contra la mujer y de desigualdad en términos laborales y educacionales; aspectos que tienen un impacto trascendente en la maternidad.

Nuestro estudio cuenta con algunas limitaciones, como el tamaño de la muestra y el tipo de muestreo; así como la falta de información sociodemográfica que permitiría hacer análisis más completos de la situación psicosocial de las mujeres puérperas. Sin embargo, dado que este es uno de los primeros estudios realizados sobre esta temática en la ciudad de Arequipa, sería necesario que en futuras investigaciones se puedan subsanar dichos aspectos, y acceder a muestras más representativas, valorando otras variables de estudio.

Se puede concluir que la depresión postparto tiene una prevalencia elevada en el distrito de Alto Selva 
Alegre, en la ciudad de Arequipa, y que el grado de instrucción se relaciona inversamente con las puntuaciones de depresión postparto.

\section{Declaración de financiamiento y de conflictos de interés:}

La investigación fue financiada por los autores. Los autores declaran que no existen conflictos de intereses.

\section{Contribución de autoría:}

MAM: planteó la idea del estudio, la recopilación de la información y de la redacción del marco teórico. WA: formuló el diseño de la metodología del estudio, procesó los resultados y redactó la discusión.

\section{Correspondencia:}

Lic. María Alejandra Salinas

Correo electrónico: maria.masias@ucsp.edu.pe

\section{REFERENCIAS BIBLIOGRÁFICAS}

1. Escolano-Pérez E. El cerebro materno y sus implicaciones en el desarrollo humano. Rev Neurol. 2013; 56(2):101-8.

2. Minchón C. Efecto de la inducción del parto con oxitocina en el Apgar bajo del recién nacido. UCV Scientia. 2009; 1(1):102-9.

3. Espíndola JG, Morales-Carmona F, Díaz E, Pimentel D, Meza, P, Henales C, Carreño J, Ibarra AC. Malestar psicológico: algunas de sus manifestaciones clínicas en la paciente gineco-obstétrica hospitalizada. Perinatol Reprod Hum. 2006; 20:112-22.

4. Jadresic E. Depresión en el embarazo y el puerperio. Rev Chil Neuro-psiquiat. 2010; 48(4):269-78.

5. Vargas-Mendoza J, García M. Depresión postparto: Presencia y manejo psicológico. Centro Regional de Investigación en Psicología. 2009; 3(1):11-8.

6. Escobar J, Pacora P, Custodio N, Villar W. Depresión posparto: ¿Se encuentra asociada a la violencia basada de género? An Fac Med. 2009; 70(2):115-8.

7. Walther V. Postpartum depression: A review for perinatal social workers. Soc. Work Health Care. 1997; 24(3-4):99-111.

8. Ibarra L. Adolescencia y maternidad. Impacto psicológico en la mujer. Rev Cuba Psicol. 2003; 20(1):43-7.

9. Olhaberry M. Interacciones tempranas y género infantil en familias monoparentales chilenas. Rev. Latinoam. Psicol. 2012; 44(2):75-86.

10. Nóblega M, Traverso P, Ugarte A, Caballero L. Factores sociodemográficos explicativos del guion de base segura materno. Rev Psicol. (Lima). 2017; 35(2):575-604.
11. Shaw BA, Krause N, Chatters LM. Emotional support from parents early in life, aging, and health. Psychology and Aging. 2004; 19(1):4-12.

12. Ayala-Nunes L, Lemos I, Nunes C. Predictores del estrés parental en madres de familias en riesgo psicosocial. Univ Psychol. 2014; 13(2):529-39.

13. González NI, Gil M. Autoestima y socialización maternal: un análisis transcultural. Persona. 2006; 9:189-201.

14. Yu R, Ho SC. Psychometric evaluation of the Perceived Stress Scale in early postmenopausal Chinese women. Psychology. 2010; 1:1-8.

15. Cerezo MA, Trenado RM, Pons-Salvador G. Interacción temprana madre-hijo y factores que afectan negativamente a la parentalidad. Psicothema. 2006; 18(3): 544-50.

16. Mitja M. Sistema de categorías para el registro de conductas interactivas precoces entre la madre y el bebé. Psicothema. 1992; 4(1):169-81.

17. Van Doorm MMEM, Kuijpers RCWM, LichtwarckAschoff A, Bodden D, Jansen M, Granic I. Does mother-child interaction mediate the relation between maternal depressive symptoms and children's mental health problems? J Child Fam Stud. 2016; 25:125768.

18. Traverso P. Dos madres adolescentes, dos vínculos: ¿qué marca la diferencia? Rev Psicol. (Lima). 2007; 25(1):59-80.

19. Runavicius R, Baptista F. Retração precoce do bebé e humor de gestantes adolescentes. Bol Acad Paulista de Psicologia. 2016; 35(89):478-507.

20. Centenaro D, Marin AH, da Silva C, Golembiewski M. ¡Llegó la hora! Expectativas y experiencias de parto de adolescentes embarazadas. Rev Psicol. (Lima). 2015; 33(2):333-61.

21. Villarán V, Traverso P, Huasasquiche ML. Narrativas sobre el embarazo y la maternidad en mujeres adolescentes de sectores urbano marginales de Lima que acaban de dar a luz. Rev Psicol. (Lima). 2017; 35(2):485-508.

22. Ato M, López JJ, Benavente A. un sistema de clasificación de los diseños de investigación en psicología. An Psicol. 2013; 29(3):1038-59.

23. Hernandez R, Fernández C, Baptista P. Metodología de la investigación. Ciudad de México: McGrawHill; 1997.

24. Lam N, Contreras H, Mori E, Hinostroza W, Hinostroza R, Torrejón E, et al. Factores psicosociales y depresión antenatal en mujeres gestantes. Estudio multicéntrico en tres hospitales de Lima, Perú. Rev Peru Epidemiol. 2010; 14(3):193-200.

25. Nóblega M. La maternidad en la vida de las adolescentes: implicancias para la acción. Rev Psicol. (Lima). 2009; 27(1):29-54.

26. Traverso P, Nóblega M. Promoviendo vínculos saludables entre madres adolescentes y sus bebés: una experiencia de intervención. Rev Psicol. (Lima). 2010; 28(2):259-83. 
27. Briolotti A, Benítez SM. Medicina, higiene mental y saber psi en la construcción de la maternidad y la infancia en la Argentina: un análisis a través de la revista Madre y Niño (1934-1935). Univ Psychol. 2014; 13(5):1709-19.

28. Winnicott DW. Conversando con los padres. Aciertos y errores en la crianza de los hijos. Barcelona: Paidós; 1993.
29. León FR. Latitud sur y control económico del hogar por la mujer peruana. Rev Psicol. (Lima). 2011; 29(2):361-88.

Recibido: 29/08/2017

Aceptado: 25/09/2018 